\title{
Labyrinthe
}

$7 \mid 2000$

Numéro 7

\section{Entre standard et création : l'hypermarché}

\section{Solange Jungers}

\section{(2) OpenEdition}

Journals

Édition électronique

URL : http://journals.openedition.org/labyrinthe/737

DOI : $10.4000 /$ labyrinthe.737

ISSN : 1950-6031

Éditeur

Hermann

Édition imprimée

Date de publication : 30 octobre 2000

Pagination : 157-160

Référence électronique

Solange Jungers, «Entre standard et création : l'hypermarché », Labyrinthe [En ligne], 7 | 2000, mis en ligne le 20 avril 2005, consulté le 19 avril 2019. URL : http://journals.openedition.org/labyrinthe/737 ; DOI : 10.4000/labyrinthe.737

Ce document a été généré automatiquement le 19 avril 2019

Propriété intellectuelle 


\title{
Entre standard et création: l'hypermarché
}

\author{
Solange Jungers
}

1 À la fin des années cinquante, la société américaine de caisses enregistreuses NCR décide de développer son marché à l'étranger, en exportant le mode de distribution auquel elle est liée : le supermarket. La grande distribution en libre service requiert en effet un nombre élevé de caisses enregistreuses modernes. Elle rencontre en France les préoccupations de nombreux épiciers qui tentent de moderniser le commerce, ainsi que les interrogations des pouvoirs publics quant à la structure commerciale à donner aux ensembles de logements récemment construits. L'expérience américaine permet aux fondateurs de Carrefour de formaliser leur concept inédit du «tout sous le même toit ». Ils empruntent d'abord les logiques commerciales du super market, l'organisation spatiale du shopping strip et la volumétrie générale du shopping center. L'invention du nouveau programme (l'hypermarché) s'accompagne de la volonté de créer une architecture commerciale nouvelle, trouvant en ses propres lois sa forme, ses matériaux, ses modes de production. De création.

2 Carrefour ouvre en 1963, à Sainte-Geneviève-des-Bois, le premier hypermarché au monde. Au cours de la maturation du projet, le maitre d'ouvrage sort de son rôle de programmation pour s'improviser maître d'œuvre; il remercie ses architectes parce qu'il souhaite maîtriser - aussi - la réalité architecturale. Il établit de fait une approche particulière de l'acte de bâtir et de créer.

3 Comme la grande distribution invente de nouvelles règles du jeu architecturales, son historien devra penser une méthode d'analyse nouvelle. En effet, la pluralité des édifices dénie à cette architecture son statut d'œuvre exclusive ; la monographie d'architecte ou de site échoue parce qu'elle isole une partie qui prend son sens dans un tout. L'historien de l'architecture commerciale devra donc emprunter ses méthodes d'analyse à l'histoire du design plutôt qu'à l'histoire de l'art. À cet élargissement correspond paradoxalement une nécessaire attention au contexte particulier de conception, auquel l'historien répondra en devenant tour à tour géographe urbain, économiste... Sans jamais oublier la 
forme et le signe, qui, s'ils ne constituent plus l'approche exclusive, restent les plus évidentes originalités - dans leur banalité ? - de l'hypermarché.

Robert Venturi, en fixant la réalité mouvante de Las Vegas au moment précis de son étude, approcha l'architecture commerciale en tant que " phénomène de communication architecturale ». En empruntant à l'histoire ses méthodes d'analyse, nous envisagerons l'hypermarché dans la durée, à travers une chronologie tenant compte des modifications. Cette approche pragmatique rejette l'idée du catalogue d'architectures extraordinaires, pour se pencher au contraire sur la production ordinaire de l'hypermarché, qui s'exhibe industriel et préfabriqué, à mi-chemin entre standard et création.

5 La production en masse d'hypermarchés ne se confond pas avec la préfabrication lourde : elle ne se réfère pas tant à la reproductibilité à l'identique de l'objet produit en industrie (fordisme) qu'à la mise au point d'un processus-type d'élaboration du projet (taylorisme). Elle tend ainsi à définir un standard. Dans les années soixante, quelques entrepreneurs en bâtiment tentent, en vain, de convaincre les distributeurs de mettre au point un prototype, conçu selon un système constructif spécifique. Tous préfèrent la construction par composants compatibles, utilisant des éléments disponibles sur le marché, qui ne soient pas liés à une opération précise. La maîtrise du projet ouvre une autre voie à la rationalisation de la construction que celle de la standardisation constructive proposée. Le standard et le plan-type s'offrent comme alternatives à l'industrialisation fermée.

6 Le discours sur l'hypermarché comme "usine à vendre " est d'abord produit par les distributeurs pour définir conceptuellement et formellement un type né directement de la construction par composants, au sein d'une logique dominée par les principes d'économie et de rentabilité. L'usine renvoie à une manière de perfection fonctionnaliste. Mais l'architecture comme "usine à vendre » fonctionnaliste introduit une confusion entre standard et type : les maîtres d'œuvre répètent la mise en espace du programme, qui recouvre une liberté créatrice dans son appropriation par le signe, la couleur, le jeu de lumières et de matières. Le bureau d'études SODETEG a ainsi réalisé une variation lumineuse cinétique sur la façade principale du Carrefour de Créteil en 1968.

7 L'analyse des maîtrises d'ouvrage permet de comprendre les raisons et les modes opératoires de ce glissement. Elle montre comment, pendant presque toute la décennie soixante, Carrefour s'est imposé comme le détenteur de la "Vérité-hypermarché », en se donnant les moyens de contrôler l'évolution du concept, notamment à travers les contrats de franchise accordés à Promodès (Continent) et aux Docks Mielle (Cora). La centrale d'achat auprès de laquelle l'hypermarché s'approvisionne, le GAGMI, contribue également à développer le modèle Carrefour, en créant un département de programmation d'hypermarchés : elle développe une véritable réflexion sur l'hypermarché-magasin et sur l'architecture, notamment en faisant montre d'un souci de régionalisation inédit, et se veut professionnelle et prospective, par le biais des conseils prodigués à ses adhérents. Mais en 1968, Carrefour se retire pour fonder sa propre centrale d'achat non alimentaire, la SAMOD. La complexité de la maitrise d'ouvrage de l'hypermarché est maintenue au sein d'une cohérence qui fait système, et réunit des coopératives (les Docks de France-Suma), des groupements d'intérêt économique (Euromarché), des associations (Leclerc), des Grandes Entreprises de Grande Surface (Carrefour, Auchan)...

8 L'hypermarché « conçoit l'architecture comme un play-boy conçoit la vie, vite blasé et courant d'une sensation à l'autre $»^{1}$. L'obsession de la contemporanéité produit une architecture nécessairement temporaire qui explique en partie la faible durée de vie des 
matériaux de construction ainsi que la légèreté du montage (boulonnage des bardages de façade par exemple). L'hypermarché exhibe sa structure temporelle particulière en une construction provisoire et une architecture éphémère.

9 Les réflexions sur la construction modulaire ou par composants compatibles qui se développent dans les années soixante (l'État homologue en 1963 une norme de coordination dimensionnelle des composants) trouvent en l'hypermarché un écho tout particulier. La normalisation dimensionnelle enrichit les possibilités combinatoires des composants issus de la standardisation ouverte, autorisant à opérer des variations à partir de modèles types, et, partant, à rejeter la voie de la standardisation fermée peu adaptée à la flexibilité nécessaire de la formule. L'élargissement du panier de composants dans les années soixante-dix sous l'impulsion de l'État (le Plan Construction) est également mis à profit par les concepteurs d'hypermarchés, qui trouvent là le moyen d'offrir de nouvelles architectures à partir d'un scénario figé (le standard).

10 Parce qu'il est éphémère par essence, l'hypermarché s'impose comme solution transitoire, établissement symbolique du centre des villes nouvelles en chantier. À Créteil comme à Montigny-le-Bretonneux (Saint-Quentin-en-Yvelines), les sociétés d'économie mixte d'aménagement ont privilégié la construction de la ville par phases, dont chacune a sa propre cohérence et sa propre viabilité. En attendant qu'un centre se mette en place, un hypermarché est construit à titre précaire pour une durée de dix ans, au terme desquels les distributeurs s'engagent à rendre le terrain en l'état (Carrefour est implanté à Créteil entre 1968 et 1978, Euromarché à Montigny-le-Bretonneux entre 1975 et 1988). À Créteil, il s'agit de trouver une solution provisoire à la difficulté d'installer un élément commercial important, dont la ville a besoin pour devenir symboliquement chef-lieu du département et s'imposer comme nouveau barycentre, alors que la mise au point du plan de zone est longue et délicate. À Montigny-le-Bretonneux, l'hypermarché est la manifestation des théories fonctionnalistes sur la nouvelle centralité développées par les urbanistes Henri Coulomb et Guy Lagneau pendant la première phase de conception de la ville, parti finalement abandonné pour revenir à la fin des années quatre-vingt à un centre « néo-traditionnel».

11 Les premières tentatives de contextualisation des hypermarchés et, plus généralement, des parcs d'activités commerciales, ont posé le problème de la gestion des «entrées de ville ", souvent considérées comme une sorte de territoire virtuel, royaume des signes ${ }^{2}$. Nous détachant de cette analyse formulée à partir d'espaces existants (traitant d'un moment), nous dressons les typologies des territoires empruntés par les hypermarchés en région parisienne (dans la durée), selon leurs propres logiques, qui, toutes, ont à voir avec la création de nouveaux barycentres, au sein de zones de chalandise créées de façon volontariste, hors des limites administratives des villes.

On pense souvent que les hypermarchés se ressemblent parce que les créateurs élaborent une architecture à partir d'un standard programmatif qui n'est pas remis en cause. L'architecture est parfois réduite à l'appropriation d'un matériau de base neutre (le standard et la construction par composants) et de normes établies par le maître d'ouvrage (trames, matériaux, couleurs déposées...). Cette acception particulière d'un acte créateur bridé ne dément pas pour autant la qualité des architectures produites, fonctionnelles, publicitaires, attirantes, mouvantes. Signifiantes. 


\section{NOTES}

1. S. Giedion, Espace, temps et architecture, tome I, Paris, Denoël/Gonthier, 1978, p. 18.

2. Se rattachent à ce courant de pensée Arnaud Sompairac à propos des stations-service (A. Sompairac, Stations-service, Paris, Centre Georges Pompidou, 1993) ou encore l'exposition «L'autre ville. L'empire des signes », tenue à l'IFA, du 25 septembre 1997 au 3 janvier 1998.

\section{AUTEUR}

\section{SOLANGE JUNGERS}

Solange Jungers est doctorante en histoire de l'architecture à l'Université Paris I. Elle prépare actuellement une thèse sous la direction de M. Gérard Monnier, sur L'Architecture des hypermarchés en Île-de-France entre 1961 et 1991, après un DEA portant sur « le système hypermarché » (1997) et une maîtrise dressant « l'inventaire des stations-service de la route Paris-Deauville, entre 1920 et $1990 »$ (1996). Allocataire-moniteur, Solange Jungers enseigne à l'Université Paris I. 